Case Report

\title{
Severe Dural Tear and Brain Injury after Vacuum Extraction Birth
}

\author{
Sebastian Senger ${ }^{1}$, Kristina Flohr ${ }^{2}$, Otto Schofer ${ }^{2}$, Joachim Oertel ${ }^{1}$, Stefan Linsler ${ }^{1^{*}}$ \\ ${ }^{1}$ Department of Neurosurgery, University Hospital of Saarland, Homburg, Germany \\ ${ }^{2}$ Department of Paediatrics , Marienhausklinik St. Josef Kohlhof, Neunkirchen, Germany
}

*Corresponding author: Linsler, S. Department of Neurosurgery, University Hospital of Saarland, Homburg, Germany. Tel: 49-6841-16-24400; Fax: 49-6841-16-24480; Email: stefan.linsler@uks.eu

\begin{abstract}
Traumatic brain lesions after vacuum extraction delivery are rare. Most common complication after operative vaginal deliveries is subgaleal hematoma applied by trained obstetricians. There are only single case reports about major complications after vacuum extraction delivery in the literature available. The authors present a case of a severe brain damage and dural tear after vacuum extraction delivery. In the presented case surgical treatment of the dural tear was required. The child recovered completely in follow up.
\end{abstract}

Keywords: Vacuum extraction birth; Brain injury; Dural tear

\author{
Received Date: May 06,2015 \\ Accepted Date: July 10, 2015 \\ Published Date: July 13, 2015
}

Citation: Senger, S. Severe Dural Tear and Brain Injury after Vacuum Extraction Birth (2015) Int J Neurol Brain Disord 2(2): 29-31.

DOI: $10.15436 / 2377-1348.15 .011$

\section{Introduction}

Delivery by vacuum extraction (VE) is a common obstetrical procedure in many countries and it has replaced the use of forceps. The use of VE has increased in the last decades rather than other methods of assisted delivery ${ }^{[1-4]}$. Vacuum extraction has become more popular because it is seemingly easy to use, requires less analgesia and is believed to be safer than other methods with modern devices ${ }^{[1]}$. While extra-cranial hematomas and skull fractures have been associated with VE assisted deliveries ${ }^{[5-7]}$, severe brain or dural injuries and intracranial bleeding ${ }^{[3]}$ are rare complications. Most common severe complication is subgaleal hematoma, resulting from rupture of the emissary veins that connect the deep dural sinuses and scalp veins. Nowadays, the modern neuroimaging techniques such as ultrasound, computer tomography (CT) and magnetic resonance imaging (MRI) have improved the diagnostic accuracy of neonatal brain damage ${ }^{[3,8]}$. But less is known about brain development and neurosurgical treatment in case of severe brain damage after VE.

\section{History and Examination}

Baby ' $\mathrm{S}$ ' was a 2980-g female infant born at 39 weeks gestation to a primigravida mother via vacuum extraction after uncomplicated pregnancy cause of prolonged birth. The baby was in an occipitoposterior position when arrest of birth occurred and cardio-tocography showed fetal distress with drops of heart sound. A VE was performed with Kiwi Omni Cup with one pull of 8 minutes duration. The head of the baby was in occipitoposterior position in mid outlet station during expulsive phase of delivery. The Apgar scores were 9/10/10 at 1, 5 and $10 \mathrm{~min}$. A large cephalic hematoma was visible directly after delivery at the frontal part. There was speculated that positioning of Kiwi Cup was more frontal than usually required.

The child was admitted to neonatal ICU for observation. She was noted to have a large growing fluctuant mass in the frontal area and suffered generalized epileptic seizure on second day after birth. She was referred to our department 3 days after delivery. At presentation, a large bi-frontal fluid collection, mimicking scaphocephaly, was visible. She was in stable condition (no hypovolemia, no brady- or tachycardia). She had no neurological deficits with normal muscle tonus and reflexes.

\section{Imaging}

Sonography revealed large subgaleal cerebrospinal fluid (CSF) accumulation and suspicion of dural tear parasagittal. On MR imaging a severe dural tear with large subgaleal CSF accumulation and also subgaleal haemorrhage was identified. Also damage of the dorsofrontal lobe and a brain prolapse was detected (Figure 1). In MR angiography, there was no evidence of injury of sagittal sinus.

Copy rights: (C2015 Linsler, S. This is an Open access article distributed under the terms of Creative Commons Attribution 4.0 International License. 

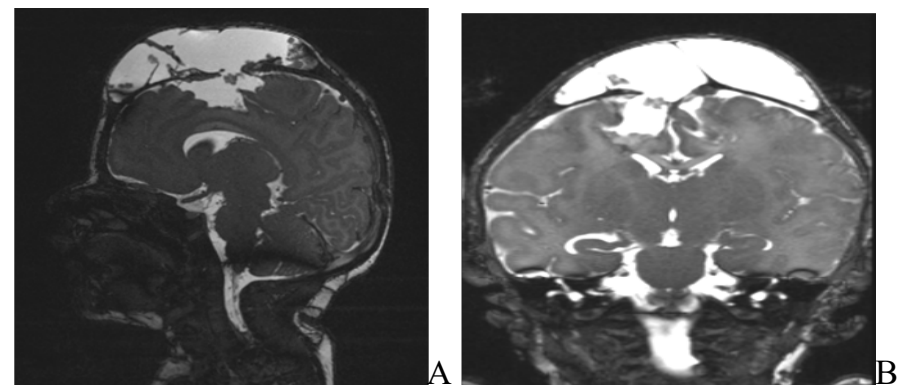

Figure 1: Showing sagittal (a) and coronal (b) MR imaging of the presented case. There is a severe dural tear right sided with large subgaleal CSF accumulation. The sagittal sinus is elevated to the left. The frontodorsal cortex of the frontal lobe is injured right-sided.

\section{Surgical Procedure and Postoperative Course}

Surgery was intended to reduce the risk of persisting CSF fistula, for inspection of the affected cortex and preventing epilepsy in follow up. Surgery was performed on day 12 after birth via a bicoronal approach. The CSF collection was surrounded by neo-membranes that were dissected to the plane of the defect around the fontanelle. The sagittal suture was ruptured. The defect of the right-side parasagittal dura was $3 \times 2.5$ $\mathrm{cm}$ (Figure 2). Intraoperatively, there was an intact sagittal sinus. The exophytic brain tissue epidural and intracerebral haemorrhage was removed. Finally human fascia lata and Tacho Sil was used for duraplasty (Figure 3). Histological testing of the extracted tissue revealed brain tissue and dura mater with evidence of old hemorrhages. Intraoperative blood loss was less than $50 \mathrm{ml}$.

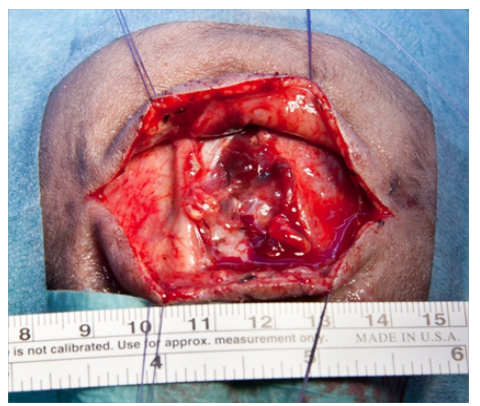

Figure 2: The statement of the MRI could be confirmed intraoperatively. The sagittal suture was ruptured. The defect of the right-side parasagittal dura was 3 $\mathrm{x} 2.5 \mathrm{~cm}$. There was an intact sagittal sinus without any bleeding.

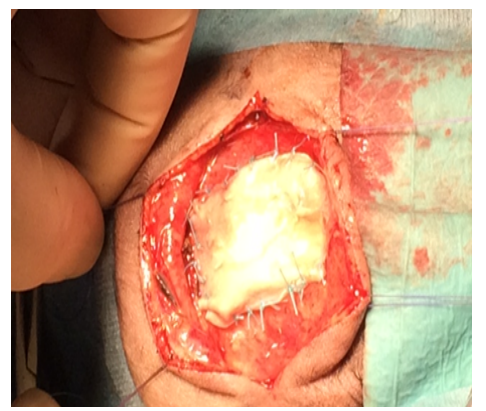

Figure 3: Shows the intraoperative results after surgical repair of the dural tear

The baby was admitted to pediatric ICU postoperatively. She had no seizures and new neurological deficits in postoperative course and was discharged at $7^{\text {th }}$ postoperative day.

At follow-up six weeks after surgery, the girl was in good conditions. She had no neurological deficits and normal development. At last follow-up after six month, the girl present in a regular development without any neurological deficits or seizures. Nevertheless, she had another large subgaleal CSF accumulation over the anterior fontanelle requiring second surgery. A posttraumatic hydrocephalus was postulated induced the second dural tear. There was a revision of the duraplasty and also fixation of an autologous bone graft performed (Figure 4). The bone graft received by craniotomy more parietal of the defect stabilized the duraplasty to prevent another dural tear cause of elevated brain pressure and brain pulsation. Additionally, an external ventricular drainage was inserted in right ventricle. Cause of posttraumatic hydrocephalus ventriculoperitoneal shunting was necessary also one week after second surgery. In last follow up, the girl was noted to have recovered completely.

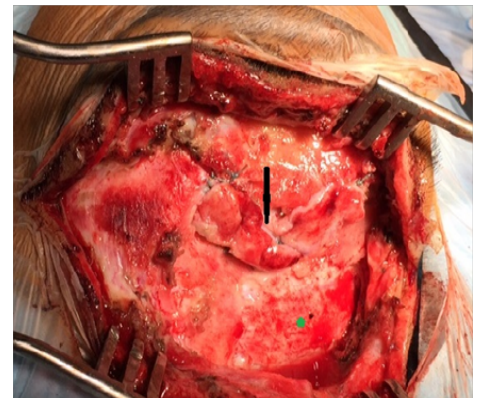

Figure 4: Shows the intraoperative results after second surgical repair of dural tear $(\mathbf{y})$ and localisation of craniotomy for the autologous bone graft $(*)$

\section{Discussion}

Vacuum delivery is a common procedure in obstetrics. In general, operative procedures for vaginal deliveries were used in $5-26 \%$ of nulliparous deliveries ${ }^{[9]}$. Historically, the vacuum is considered the instrument of choice due to a posited reduction in maternal injuries, although long-term follow-up suggests no difference in pelvic floor function between women who have undergone vacuum delivery and those who have had forceps delivery ${ }^{[10,11]}$. In a 1979 review of 15 studies involving 7124 infants who were delivered by vacuum extraction with the metal Malmstrom cup, the incidence of ICH was 1 in 286 infants $^{[12]}$. This rate decreased after the introduction of plastic cups, to an incidence of $1 \%$ reported in $1999^{[13]}$. Compared with those born by spontaneous vaginal delivery, infants delivered by vacuum extraction have significantly higher rates of subdural or cerebral hemorrhage, brachial plexus injury, and seizures ${ }^{[3,13]}$. Towner et al. ${ }^{[13]}$ reported ICH in 1 of every 860 infants delivered by vacuum extraction.

Nevertheless, fetal weight and head position should be evaluated carefully before operative vaginal delivery is undertaken to minimize the risk of bad fetal outcome ${ }^{[1,14]}$. The spectrum of possible sequelae after vacuum extraction is broad, and includes circular fracture; elevation of the outer table of skull bones; subperiosteal, intraosseous, subdural, or tentorial hematomas; and intra-cerebral and intra-ventricular haemorrhaging ${ }^{[8,15-17]}$. The vertical stress may lead to laceration of bridging veins and venous sinuses, and venous hemorrhagic infarctions ${ }^{[17]}$. The most common complication is subgaleal hematomas, which resolve on their own in the majority of $\operatorname{cases}^{[18]}$. However, Amar et al. ${ }^{[15]}$ reported on 2 cases of subgaleal hematomas causing brain compression, which required surgical intervention to control elevated intracranial pressure. Factors associated with a significant 
increase in subgaleal hemorrhage include: nulliparity, failed VE, presentation in an occipito-lateral or occipito-posterior position, marks of vacuum cup over the sagittal suture or the leading edge of vacuum cup at $<3 \mathrm{~cm}$ away from the anterior fontanel, and an Apgar score of less than eight at $5 \mathrm{~min}^{[19,20]}$. Close observation of infants born by VE with such risk factors should be observed closely for the development of a subgaleal hemorrhage ${ }^{[19]}$.

Sporadic reports exist in the literature on leptomeningeal cysts caused by vacuum extraction with dural tear, predominantly located over the anterior fontanelle and rupture of the anterior fontanelle and the sagittal suture ${ }^{[14,21]}$. The pattern of injury in our patient is similar to these reports and supports the hypothesis that laceration of the dura and brain tissue may be a factor in some cases of persistent, massive subgaleal fluid accumulation. The suction of vacuum extraction delivery is strong enough to induce aspiration brain tissue after severe dural tears. Thereby wrong positioning of the cup over the anterior fontanelle may improve the risk of severe dural tears.

Long-term follow-up of babies born via vacuum extraction who experienced brain damage usually shows complete resolution of hemorrhage and show a regular neurological development ${ }^{[14]}$ as presented in this case. Surgery is only required in rare cases of severe dural ruptures preventing persisting CSF fistulas. In case of severe dural tears and possible posttraumatic hydrocephalus bone graft and external ventricular drainage or ventriculoperitoneal shunting might be necessary additional to duraplasty.

The limitation of this report is the slight neurosurgical experience with TBI after obstetrical procedures as presented in this case. There is only one comparable case report in the literature available. But on account of this, the presented case report might draw this knowledge gap in the literature and provide a basis of further neurosurgical treatment in comparable cases.

\section{Acknowledgement}

All authors certify that they have no other affiliations with or involvement in any organization or entity with any financial interest or non-financial interest in the subject matter or materials discussed in this manuscript.

\section{References}

1. Johanson, R.B., Menon, B.K. Vacuum extraction versus forceps for assisted vaginal delivery. (2000) Cochrane Database Syst Rev (2): CD000224.

2. Kozak, L.J., Weeks, J.D. U.S. trends in obstetric procedures, 19902000. (2002) Birth 29(3): 157-161.
3. Ekeus, C., Hogberg, U., Norman, M. Vacuum assisted birth and risk for cerebral complications in term newborn infants: a population-based cohort study. (2014) BMC Pregnancy Childbirth 14: 36.

4. Kicklighter, S.D., Wolfe, D., Perciaccante, J.V. Subgaleal hemorrhage with dural tear and parietal-lobe herniation in association with a vacuum extraction. (2007) J Perinatol 27(12): 797-799.

5. Doumouchtsis, S.K. Arulkumaran, S. Head injuries after instrumental vaginal deliveries. (2006) Curr Opin Obstet Gynecol 18(2): 129-134.

6. Doumouchtsis, S.K., Arulkumaran, S. Head trauma after instrumental births. (2008) Clin Perinatol 35(1): 69-83.

7. Swanson, A.E., Veldman, A., Wallace, E.M., et al. Subgaleal hemorrhage: risk factors and outcomes. (2012) Acta Obstet Gynecol Scand 91(2): 260-263.

8. Odita, J.C., Hebi, S. CT and MRI characteristics of intracranial hemorrhage complicating breech and vacuum delivery. (1996) Pediatr Radiol 26(11): 782-785.

9. Drife, J.O. Choice and instrumental delivery. (1996) Br J Obstet Gynaecol 103(7): 608-611.

10. Baskett, T.F. Epidemiology of obstetric critical care. (2008) Best Pract Res Clin Obstet Gynaecol 22(5): 763-774.

11. Baskett, T.F., Fanning, C.A.,Young, D.C. A prospective observational study of 1000 vacuum assisted deliveries with the OmniCup device. (2008) J Obstet Gynaecol Can 30(7): 573-580.

12. Plauche, W.C. Fetal cranial injuries related to delivery with the Malmstrom vacuum extractor. (1979) Obstet Gynecol 53(6): 750-757.

13. Towner, D., Castro, M.A., Eby-Wilkens, E., et al. Effect of mode of delivery in nulliparous women on neonatal intracranial injury. (1999) N Engl J Med 341(23): 1709-1714.

14. Musahl, C., Schick, U. Severe brain injury with rupture of the superior sagittal sinus after vacuum extraction birth. (2008) J Neurosurg Pediatr 1(6): 471-473.

15. Amar, A.P., Aryan, H.E., Meltzer, H.S., et al. Neonatal subgaleal hematoma causing brain compression: report of two cases and review of the literature. (2003) Neurosurgery 52(6): 1470-1474.

16. Hanigan, W.C., Morgan, A.M., Stahlberg, L.K., et al. Tentorial hemorrhage associated with vacuum extraction. (1990) Pediatrics 85(4): 534-539.

17. Rupp, W., Ropohl, D., Bohnert, M. [Differential diagnosis of traumatic skull findings in infants: residues after vacuum extraction]. (2005) Arch Kriminol 215(3-4): 70-76.

18. Ryan, T.D., Dimmitt, R.A., Tubbs, R.S., et al. Contribution of surgical evacuation of a large subgaleal hematoma to the resolution of severe conjugated hyperbilirubinemia in a neonate. Case report (2007) J Neurosurg 106(2 Suppl):131-133.

19. Walsh, C.A., Robson, M., McAuliffe, F.M. Mode of delivery at term and adverse neonatal outcomes. (2013) Obstet Gynecol 121(1): 122-128.

20. Whitby, E.H., Griffiths, P.D., Rutter, S., et al. Frequency and natural history of subdural haemorrhages in babies and relation to obstetric factors. (2004) Lancet 363(9412): 846-851.

21. Kau, T., Scharfegger, H., Gasser, J., et al. Pseudomeningocele and traumatic brain lesion following vacuum extraction delivery. (2007) Arch Neurol 64(7): 1048-1049.
Online ISSN: 2377-1348

Journal Title: International Journal Neurology and Brain Disorders Journal Short Name: Int J Neurol Brain Disord
Ommega Online Publishers

E-mail: neurology@ommegaonline.com

Website: www.ommegaonline.com 